\title{
inCrease: an Approach for Particle Enhanced Soft Composite 3D-Printed Tactile Displays
}

\author{
Marc van Almkerk \\ University of Twente \\ The Netherlands \\ m.vanalmkerk \\ @student.utwente.nl
}

\author{
Casper Kessels \\ University of Twente \\ The Netherlands \\ c.g.a.kessels \\ @student.utwente.nl
}

\author{
Gijs Huisman \\ University of Twente \\ The Netherlands \\ gijs.huisman \\ @utwente.nl
}

\begin{abstract}
We present ongoing work in the development of a novel method for providing tactile feedback by using 3D-printed particle-enhanced soft composites (PESC) that, when compressed, produce a creased surface. By controlling the creasing of the material through 3D-printed rigid structures embedded in the material various textures on the material's surface can be produced. We show, through several iterations, the impact of different 3D-printed designs on the production of textures We discuss limitations and highlight possible application areas in $\mathrm{HCl}$ for the proposed approach.
\end{abstract}

Haptic. Tactile display. Texture. 3D-printing.

\section{INTRODUCTION}

Humans are capable of detecting changes in the texture of a surface that are only a few nanometers high [19]. This incredible sensitivity allows us to feel the difference between burlap, cotton and silk, or the goosebumps on the back of a lover's neck during a loving embrace.

Despite the sensitivity of the human sense of touch, conventional interface technologies in HumanComputer Interaction $(\mathrm{HCl})$ primarily focus on interactions on the smooth glass surfaces of touch screens. Considering the capabilities of the human sense of touch, the addition of adjustable textures to these types of interfaces could provide richer information to users and could enable novel interaction paradigms.

Here, we present ongoing work on the development of a novel approach to tactile displays that utilizes 3D-printed particle-enhanced soft composites (PESC) to produce creases in the material's surface [7] in order to create a controllable textured surface. We refer to this approach as inCrease. In the following sections, we will discuss related work, present the design approach, as well as several iterations of 3D printed designs, and propose future applications for $\mathrm{HCl}$ research.

\section{RELATED WORK}

In $\mathrm{HCl}$ research there have been several efforts to add tactile information in the form of textures to computer interfaces, for example, by enhancing touch screens with tactile overlays [4], or through stylus or finger input devices that provide tactile feedback [17][18]. Similar approaches have been used to enhance tactile sensations on physical objects in augmented reality [3]. All of these approaches use vibrations to produce tactile texture sensations. This is in contrast to shape changing interfaces that physically alter their shape to provide a user with tactile feedback [8][16]. In one approach, 'physical displays' use individually addressable 'physical pixels' to provide users with tactile information [16], including texture-based information [8]. For example, inForm uses linear actuators to move individual pins in an array [6]. Lee et al. [11] present a similar approach using a high-resolution pin array to provide texture information. Other approaches include the use of smart materials, such as nitinol shape-memory alloy, to produce soft, pliable physical pixels that can be individually addressed [5][13][15]. Individually controllable physical pixels can provide rich information to users, but they require each pixel to be controlled by an actuator, creating complex setups in terms of necessary control hardware.

Researchers have addressed this complexity by looking at either simplified pin arrays, taking for granted a lower resolution [2][9][14], or by looking at properties of pliable materials that produce textures when their physical state is changed [8][9]. Harrison et al. [8] demonstrated that the texture of materials, such as woven elastic, spandex, and latex, under stress (i.e., when stretched) differs from the texture of the materials in a relaxed state. Building on this idea, Hoggan et al. [9] used compression of mylar 
sheets to produce diamond-like textures in a cylindrical shape. One potential limitation of these approaches is that off-the-shelf materials are used which offer relatively little control over the variety and types of textures that can be produced by physical deformation. inCrease is in line with these last two approaches but utilizes 3D-printing of flexible and rigid materials to control the topography of the surface textures produced as a function of the physical deformation of the material.

\section{DESIGN APPROACH OF INCREASE}

inCrease builds on the approaches introduced by Guttag and Boyce [7], who proposed PESC designs that consist of flexible materials that are supplemented with relatively hard materials, and by Liao et al. [12], who suggested the use of flexible materials that contain cavities. Both approaches are based on the idea of integrating a matrix of either hard material or cavities inside a flexible substrate that allows for changes in surface topography under compression (Figure 1). By varying the inner matrix of these PESCs, different surface topographies can be achieved. In our work, we build upon these ideas by materializing them through $3 \mathrm{D}$ printing technology and we explore how these ideas can be utilized through a physical and applied approach as tactile feedback in human-computer interaction. The PESC specimen as designed for inCrease are 3D-printed using an Objet260 Connex3 that allows printing in both flexible material, called "Tango", and rigid material, "Vero", based on the Polyjet printing technique that enables high-resolution prints [20]. This specific printing technique enabled us to print the special geometry involved in these PESC designs.

\subsection{Design explorations of 3D-printed PESCs}

A first set of prints was made containing rigid material in different shapes, inter-particle spacing and rotation, embedded within a soft material (Figure 2). When compressed these prints did show differential effects of the shapes of the rigid material on the surface topography of the flexible material. In addition, it was observed that the sides of the print also produced a well-pronounced texture (Figure 3a), an effect not discussed in previous work [7]. Because of the unexpected nature of this effect, it was decided to explore this further, as a potential alternative approach. Another print was made in which the rigid material was oriented parallel to the surface of the flexible material (Figure $3 \mathrm{~b}$, top). This print had a size of $100(\mathrm{l}) \times 36(\mathrm{w}) \times 8(\mathrm{~h}) \mathrm{mm}$ and a $4.32 \mathrm{~mm}$ inter-particle spacing for the rigid material. Upon compression of this print, the flexible material is pushed around the rigid material creating relatively well-defined textures due to the combination of flexible and rigid material that can be

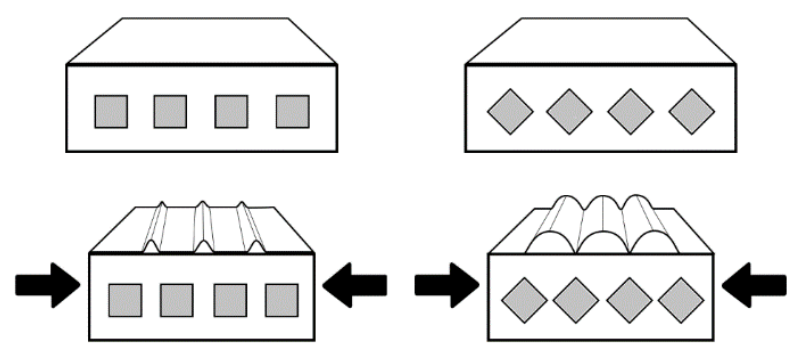

Figure 1: Simplified schematic drawing of the inCrease tactile pixel. Grey represents rigid material, while white is flexible material. Arrows represent force applied on the sides of the PESC and show that different shapes result in different surface topography.

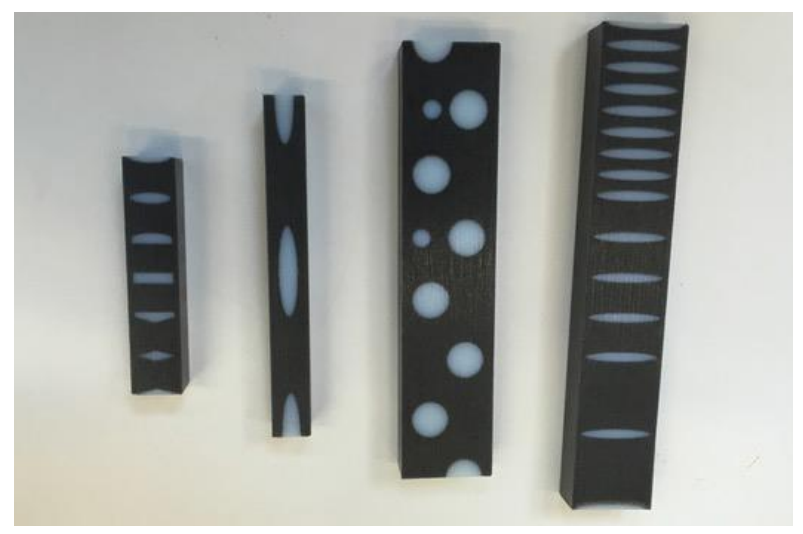

Figure 2: First set of prints with different shapes, interparticle spacing and rotation.

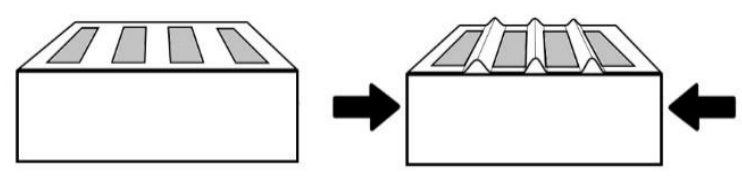

(a)

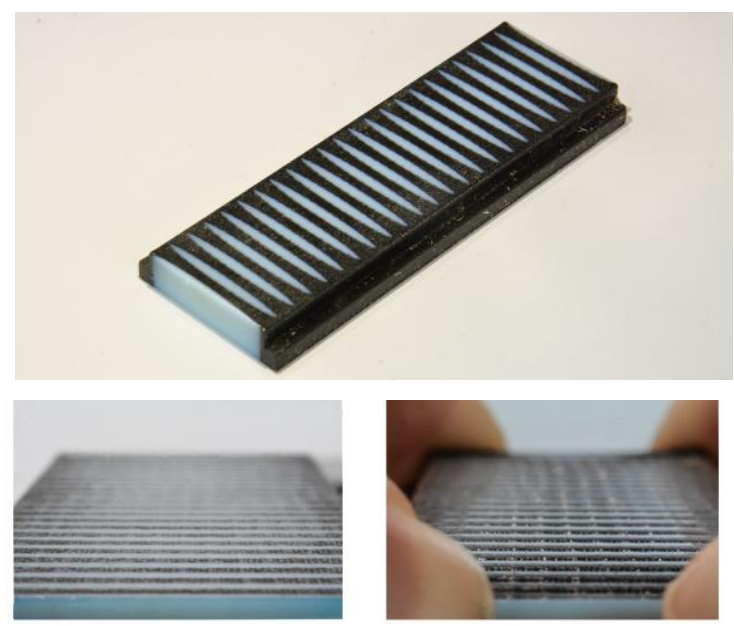

(b)

Figure 3: (a) Alternative way of using the PESC designs to produce topographic to the surface by rotating the PESC 90 degrees from its central axis. (b) physical PESC design exploring this principle. 
felt (Figure 3b, bottom). A downside of this alternative approach was that a relatively high compression force was necessary to make the textures be felt.

Based on these initial tests, a second exploration was conducted, inspired by the work of Liao et al. [12], in which two prints were made that featured rigid materials combined with cavities. Firstly to reduce the force necessary for compression, and secondly to aid in the forming of more pronounced textures. Differently shaped cavities were designed and embedded in a 3D print with a size of $100(\mathrm{l}) \times 30(\mathrm{w}) \times 10$ (h)mm (Figure 4). Moreover, to see how the surface topography changes with different inter-particle spacings, this parameter varied between 4.33 to $15.4 \mathrm{~mm}$. It was found that the prints were indeed more easily compressed and produced clear textures. Two downsides were also discovered: the produced texture was less stable (e.g. material could move around within the cavities) and the prints tore more easily after repeated compressions. Insights from these first explorations were used to create a proposed design for inCrease that was again more closely related to the work of Guttag and Boyce [7].

\subsection{Proposed design of inCrease}

Different prints were made to more clearly demonstrate how PESC prints could be used to create highly controllable tactile textures. First, two small prints were made with a size of 49(l)x12(w)x12(h)mm: one with a matrix containing a rectangular shape, and the other with a diamond shape with the same inter-particle spacing of 9.75 $\mathrm{mm}$. Both prints show a flat surface when not compressed (Figure 5, top). Under compression, however, the prints both produce distinct surface topographies. The print with embedded rectangular shapes produces ridge-like creases on the surface with relatively flat parts in between (Figure 5, bottom left), whereas the print with embedded diamond shapes produces flowing, valley-like creases on the surface (Figure 5, bottom right). For both prints, fully compressing the print (i.e. compressing the prints to the point where they start to bend) resulted in a peak difference of $2 \mathrm{~mm}$ on the surface of the print. A kitchen scale (Salter Air Super Slim) was used to get a rough estimate of the amount of force required to reach full compression of the prints. Approximately $22.55 \mathrm{~N}$ (applying 2300 grams to the top of the print) was required for full compression.

Based on the design of these two small prints, two larger square prints were created with a size of $49(\mathrm{l}) \times 49(\mathrm{~b}) \times 13(\mathrm{~h}) \mathrm{mm}$ and the same inter-particle spacing, that were more suitable as 'physical pixels' due to their shape and size. Figure 6 show that these larger prints produce creased surfaces similar to the smaller prints (i.e. $2 \mathrm{~mm}$ change on the surface of the

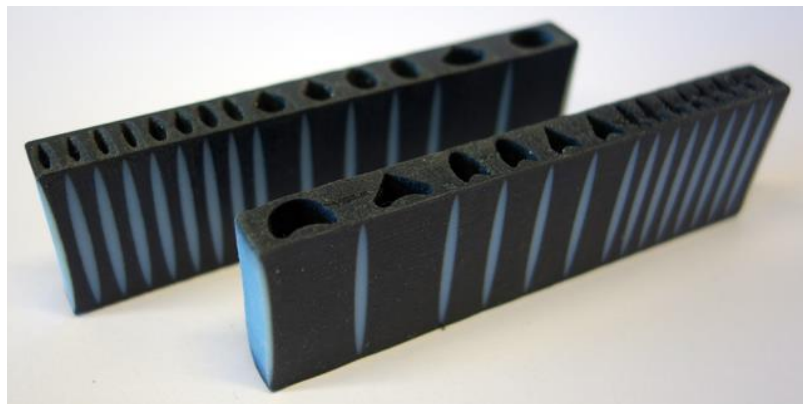

Figure 4: Iteration with cavities in different shapes.

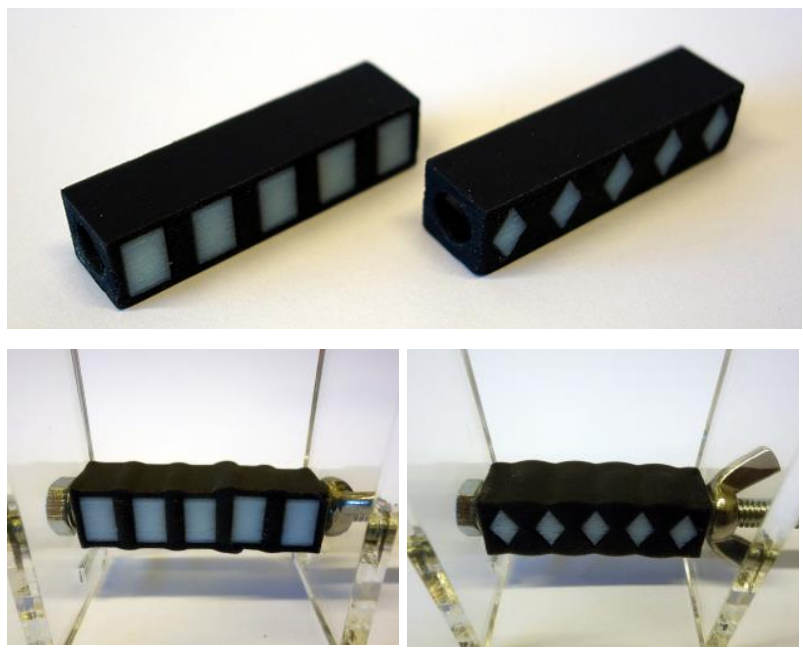

Figure 5: Small prints with rectangular and diamond pattern (top). Compressed prints showing ridge-like and valley-like creases respectively (bottom).
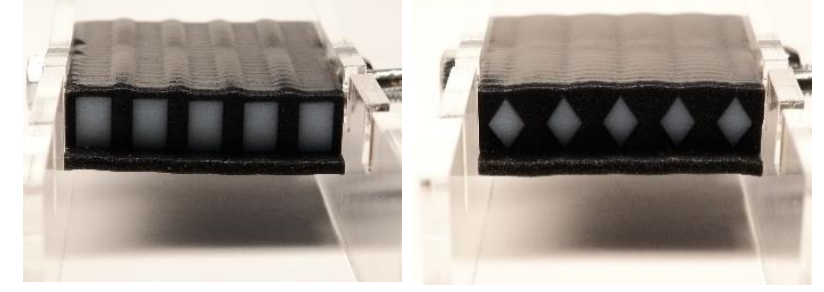

Figure 6: The creases produced by bigger prints deliver the same result as the small prints.

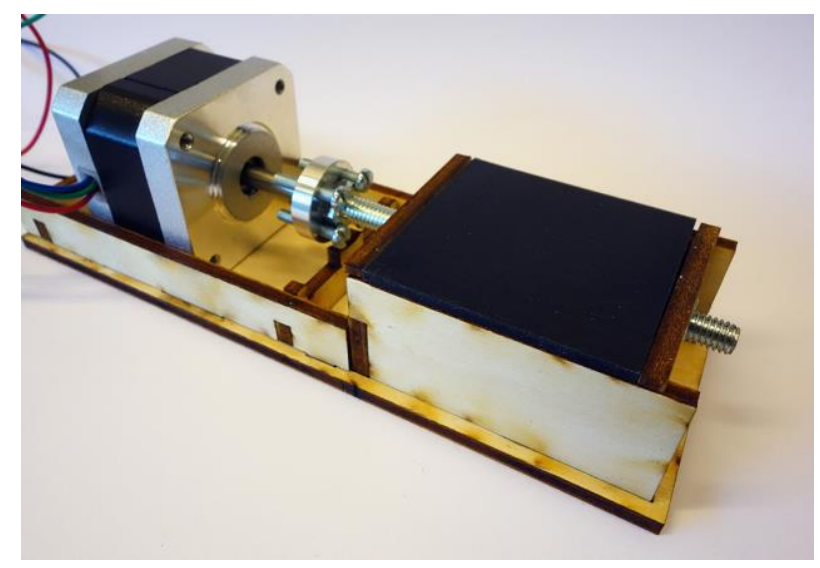

Figure 7: Prototype of a compression mechanism that can be used to test and dynamically compress PESC designs. 
print). However, these larger prints require considerably more force to reach full compression ( 47.07 N / 4800 grams).

Both the small and square prints were designed to be compressed using an off-the-shelf screw and nut that ran through a hole in each print. Like a vise, tightening the screw compresses the print. Based on this principle an initial design was made for computer controllable compression mechanism. (Figure 7). The mechanism consists of a screw that is attached to a Wantai stepper motor (42BY GHW811) secured to the base of the mechanism. The motor is controlled by a DRV8825 stepper motor driver which allows for accurate control of the motor's rotation. The stepper motor's holding torque was enough to compress the large square prints. A print can slide over the screw against a secured plate and when the axle rotates, a second plate moves along the axle compressing the print, again, like a vise. The compression mechanism thus allows for the compression of a single 'physical pixel' which creases depending on the force of compression producing a textured surface. This computercontrolled mechanism allows for interesting applications of inCrease in $\mathrm{HCl}$.

\section{Conclusions AND Discussion}

We have presented work on the exploration of PESC 3D-printing as a design approach to creating a texture display called inCrease. In an exploratory approach, the possibilities and limitations of different designs of PESCs were investigated. Explorations with the various prints show that the inCrease technique has potential as a tactile display to be used in a variety of settings. With this in mind, some recommendations for future research can be made.

Special attention has to be paid to the size of the prints. Prints of a larger size, such as the square prints presented here (Figure 6), do offer more surface area to create textures on and be touched by a user, but this comes at the cost of requiring higher compression forces. A way to reduce the necessary compression force is by creating cavities in the prints. The explorations presented here show the promise of this technique but one has to be mindful that the prints are overall less stable when compressed (i.e. they tend to flex), produce less consistent textures, and are more prone to tearing. Future research could focus on finding the optimal balance between the size of prints and the size of cavities in order to create more easily compressible yet stable prints that produce consistent textures. In addition, the explorations here demonstrate that not only the top surface of the print but also the sides of the print could be utilized to display different textures. An advantage of this latter approach is that the user touches both rigid and flexible materials which may provide additional opportunities for variations in the texture.
Important to the design of specific prints is the context in which they may be used. By using a computer-controlled compression mechanism, of which a first design is presented here, prints can be manipulated dynamically. This offers opportunities for various applications in $\mathrm{HCl}$. For example, eyesfree interfaces can be created that can offer benefits in situations where the use of visual and/or auditory feedback is restricted, or where visual and/or auditory channels are overloaded. Texture feedback could be given on the back of a mobile device (embedded in the device, or in a cover for the device) to provide users with persistent information when the sound of the phone is turned off. Here, prints of a small size would be most viable. Another example of such an application would be in automotive interfaces for drivers that could be distracted by other forms of feedback. This offers the opportunity of a larger surface area and could provide the driver with information (e.g. lane departure warning) by embedding 3D printed structures in the steering wheel. Future applications can be conceived for autonomous vehicles where inCrease structures could be embedded in the surface of the dashboard or seats providing information about the cars behavior (e.g. changes in direction or speed).

A different application area would be in interactions with interactive robotic objects potentially in combination with VR. Snake Charmer, presented by Araujo et al. [1], shows how a robotic arm could present different textures to a user in VR. Embedding inCrease in such a system could enhance the range of textures that can be presented to users. Related to this, inCrease could also be embedded into the 'skin' of social robots. This would enable interesting social touch interaction possibilities between a robot and a human by having the robot emulate human-like behavior (e.g. robotic goosebumps to indicate a robot's affective response) [12].

The current version of inCrease is not yet suitable for these applications but can serve for evaluation purposes. Such evaluations could focus on perceptual aspects of the textures at different compression rates (e.g. least noticeable differences). In addition, participants could be presented with prints with different embedded structures to see if they can distinguish the textures clearly. User evaluations would be a logical next step in validating the potential of inCrease. For now, PESC 3D-printing, as applied in inCrease, offers a novel and promising way of providing tactile texture feedback to users and could be a valuable additional technique for current tactile texture displays and shape changing interfaces. 


\section{ACKNOWLEDGEMENTS}

Special thanks to Christian Willemse, Meike Berkhoff, Sophie de Haan and Nicola Marcon for their assistance in the early stages of development. This publication is supported by the 4TU Centre for Humans \& Technology.

\section{REFERENCES}

[1] Araujo, B., Jota, R., Perumal, V., Yao, J. X., Singh, K., \& Wigdor, D. (2016, February). Snake Charmer: Physically Enabling Virtual Objects. In Proceedings of the TEl'16: Tenth International Conference on Tangible, Embedded, and Embodied Interaction (pp. 218-226). ACM.

[2] Bau, O., Petrevski, U., \& Mackay, W. (2009). BubbleWrap: a textile-based electromagnetic haptic display. In $\mathrm{CHI} 09$ Extended Abstracts on Human Factors in Computing Systems (pp. 3607-3612). ACM.

[3] Bau, O., \& Poupyrev, I. (2012). REVEL: tactile feedback technology for augmented reality. ACM Transactions on Graphics (TOG), 31(4), 89.

[4] Bau, O., Poupyrev, I., Israr, A., \& Harrison, C. (2010). TeslaTouch: electrovibration for touch surfaces. In Proceedings of the 23nd annual ACM symposium on User interface software and technology (pp. 283-292). ACM.

[5] Coelho, M., Ishii, H., \& Maes, P. (2008). Surflex: a programmable surface for the design of tangible interfaces. In $\mathrm{CHI} 08$ extended abstracts on Human factors in computing systems (pp. 3429-3434). ACM.

[6] Follmer, S., Leithinger, D., Olwal, A., Hogge, A., \& Ishii, H. (2013). inFORM: dynamic physical affordances and constraints through shape and object actuation. In Uist (Vol. 13, pp. 417-426).

[7] Guttag, M., \& Boyce, M. C. (2015). Locally and Dynamically Controllable Surface Topography Through the Use of Particle-Enhanced Soft Composites. Advanced Functional Materials, 25(24), 3641-3647.

[8] Harrison, C., \& Hudson, S. E. (2009). Texture displays: a passive approach to tactile presentation. In Proceedings of the SIGCHI Conference on Human Factors in Computing Systems (pp. 2261-2264). ACM.

[9] Hoggan, E., Hsieh, Y.-T., Myllymaa, K., Lantz, V., Kildal, J., Eiler, J., \& Jacucci, G. (2017). An Exploration of Mobile Shape-Changing Textures. In Proceedings of the Tenth International Conference on Tangible, Embedded, and Embodied Interaction (pp. 275-282). ACM.
[10] Huisman, G. (2017). Social touch technology: A survey of haptic technology for social touch. IEEE transactions on haptics, 10(3), 391-408.

[11] Lee, J. M., Wagner, C. R., Lederman, S. J., \& Howe, R. D. (2003). Spatial low pass filters for pin actuated tactile displays. In Haptic Interfaces for Virtual Environment and Teleoperator Systems, 2003. HAPTICS 2003. Proceedings. 11th Symposium on (pp. 57-62). IEEE.

[12] Liao, X., Zhu, L., Xiao, H., Pan, J., Hao, F., Shi, X., \& Chen, X. (2017). Unconventional Localization Prior to Wrinkles and Controllable Surface Patterns of Film Substrate Bilayers Through Patterned Defects in Substrate. ArXiv Preprint ArXiv:1703.10078.

[13] Nojima, T., Ooide, Y., \& Kawaguchi, H. (2013). Hairlytop interface: An interactive surface display comprised of hair-like soft actuators. In World Haptics Conference (WHC), 2013 (pp. 431-435). IEEE.

[14] Park, Y.-W., Bae, S.-H., \& Nam, T.-J. (2012). How do couples use cheektouch over phone calls? In Proceedings of the SIGCHI Conference on Human Factors in Computing Systems (pp. 763-766). ACM.

[15] Raffle, H., Tichenor, J., \& Ishii, H. (2004). Super Cilia skin: A textural interface. Textile, 2(3), 328-347.

[16] Rasmussen, M. K., Pedersen, E. W., Petersen, M. G., \& Hornbla ek, K. (2012). Shape-changing interfaces: a review of the design space and open research questions. In Proceedings of the SIGCHI Conference on Human Factors in Computing Systems (pp. 735-744). ACM.

[17] Romano, J. M., \& Kuchenbecker, K. J. (2012). Creating realistic virtual textures from contact acceleration data. IEEE Transactions on Haptics, 5(2), 109-119.

[18] Saga, S., \& Raskar, R. (2013). Simultaneous geometry and texture display based on lateral force for touchscreen. In World Haptics Conference (WHC), 2013 (pp. 437-442). IEEE.

[19] Skedung, L., Arvidsson, M., Chung, J. Y., Stafford, C. M., Berglund, B., \& Rutland, M. W. (2013). Feeling small: exploring the tactile perception limits. Scientific Reports, 3, 2617.

[20] Wong, K. V., \& Hernandez, A. (2012). A review of additive manufacturing. ISRN Mechanical Engineering, 2012. 